BIO Web of Conferences 2, 03007 (2014)

DOI: $10.1051 /$ bioconf/ 20140203007

(C) Owned by the authors, published by EDP Sciences, 2014

\title{
Synthesis of analogues of cometary organic matter: thermo- chemical evolution and preparation of in-situ observations
}

\author{
N.Fray ${ }^{1}$, G. Briani ${ }^{1,2}$, L. Le Roy ${ }^{1,3}$, Y. Bénilan ${ }^{1}$, H. Cottin ${ }^{1} \&$ M.-C. Gazeau ${ }^{1}$ \\ ${ }^{1}$ LISA, Laboratoire Interuniversitaire des Systèmes Atmosphériques, UMR 7583 du CNRS, \\ Université Paris-Est Créteil (UPEC) et Université Paris-Diderot (UPD), 94000 Créteil, France \\ ${ }^{2}$ CSNSM, Centre de Spectrométrie Nucléaire et de Spectrométrie de Masse, UMR 8609 du CNRS, \\ Université Paris Sud, 91405 Orsay, France \\ ${ }^{3}$ PhysikalischesInstitut, University of Bern, Sidlerstrasse 5, 3012 Bern, Switzerland
}

\begin{abstract}
An organic residue is remaining at $300 \mathrm{~K}$ after the VUV photolysis of ices mixture and subsequent heating. This residue is thought to be representative of the organic matter contained in comets. Our experiments show that the thermal evolution is an important process. Indeed, the organic residue remaining at $300 \mathrm{~K}$ is not produced completely during the photolysis at low temperature but also during the heating. Furthermore, when heated at temperature higher than $300 \mathrm{~K}$, the residue undergoes chemical evolution which has to be taken into account in astrophysical models. Furthermore, our work allows to propose observational strategy to maximize the chance to detect in-situ some compounds, such as POM and HMT, thanks to COSIMA which is one of the mass spectrometers on board of the Rosetta spacecraft.
\end{abstract}

\section{Introduction}

The in-situ mass spectrometry analyses, mainly those performed in 1986 in the coma of comet $1 \mathrm{P} /$ Halley, have revealed the undisputed presence of solid organic matter of large molecular weight in cometary grains. Some grains being composed only of organic matter made of carbon, hydrogen, oxygen and nitrogen atoms, they have been called "CHON grains". Nevertheless, only little information, as the elemental ratios, has been obtained and the exact nature of the cometary organic matter is still unclear.

Nevertheless, analogues of cometary organic matter can be synthesised in laboratory thanks to VUV photolysis and slow heating of ices mixtures. These energy depositions on icy films should be representative of the evolution of grain mantles of ices in molecular clouds and YSOs (Young Stellar Objects). We have developed an experimental setup allowing preparation of ice films and VUV photolysis at low temperature (about $20 \mathrm{~K}$ ). After VUV photolysis, the solid sample can be heated slowly leaving an organic residue at about $300 \mathrm{~K}$. The thermal evolution of this organic residue can be followed up to $800 \mathrm{~K}$. These high temperatures are representatives of those reached by the smallest cometary grains at $1 \mathrm{AU}$ from the Sun, and this very large temperature range, from $20 \mathrm{~K}$ to $800 \mathrm{~K}$ is the major originality of the experimental setup that we have developed. During the whole duration of each experiment, the solid and gaseous phase are characterised continuously by transmission infrared spectroscopy and mass spectrometry respectively. This allows us to correlate the thermal evolution of solid and gas phases. 


\section{Polyoxymethylene}

The Polyoxymethylene, also called POM, is a formaldehyde $\left(\mathrm{H}_{2} \mathrm{CO}\right)$ polymer. It has already been shown that POM could be synthesised from a $\mathrm{H}_{2} \mathrm{CO}: \mathrm{NH}_{3}$ ices mixture in astrophysical conditions [1]. Moreover, the presence of POM in cometary grains and its thermal decomposition in gaseous $\mathrm{H}_{2} \mathrm{CO}$ is a viable hypothesis to explain the $\mathrm{H}_{2} \mathrm{CO}$ distributed source observed in numerous comets [2-3].Thus, the presence of POM is likely even if this compound has never been clearly detected in cometary grains.

We have performed new syntheses of POM from $\mathrm{H}_{2} \mathrm{CO}: \mathrm{NH}_{3}(1: 2)$ ices mixture [4]. First, we have followed the thermal decomposition at constant temperature on very long time (several days for some experiments). It allows a very good determination of the temporal evolution of POM. Furthermore, some samples have been analysed with a ground model of COSIMA (Time of Flight Secondary Ions Mass Spectrometer on board of the Rosetta mission). These mass spectra show that the specific signature of POM is an alternation of peaks with separation of 30.011 amu which correspond to the loss of neutral $\mathrm{H}_{2} \mathrm{CO}$ during the fragmentation process. Thanks to this work, an operational strategy has been proposed to maximize the chance to detect POM in-situ thanks to COSIMA instrument.

\section{Hexamethylenetetramine}

Hexamethylenetetramine, also called HMT $\left(\mathrm{C}_{6} \mathrm{H}_{12} \mathrm{~N}_{4}\right)$, has already been identified by numerous authors and several analytical techniques in organic residues remaining at $300 \mathrm{~K}$ after VUV photolysis of ices mixtures and subsequent heating at 300K [5-6]. Recently, the precise chemical mechanism of HMT formation has been revealed by the PIIM team [7]. In collaboration with this team, we have identity, for the first time, several chemical intermediates $\left(\mathrm{NH}_{2} \mathrm{CH}_{2} \mathrm{OH}\right.$ and TMT) in a sample produced by the VUV photolysis of a $\mathrm{H}_{2} \mathrm{O}: \mathrm{CH}_{3} \mathrm{OH}: \mathrm{NH}_{3}$ ices mixtures. This work reveals clearly that the organic residue remaining at $300 \mathrm{~K}$ is not only produced during the VUV photolysis step at low temperature but also during the subsequent; it is in particular the case of HMT [8]. Indeed, the last reactions leading to the HMT synthesis have large activation barriers and need to be thermally activated. We have also studied the thermal evolution of organic residues containing HMT up to $550 \mathrm{~K}$. We show that from $350 \mathrm{~K}$ HMT sublimates quite rapidly with a kinetic rate which depends of the composition of the residue [9]. As HMT sublimates quite easily, in the frame of the Rosetta mission, this compounds has to be searched in solid as well as in gas phase thanks to COSIMA and ROSINA respectively.

\section{References}

1 W. A. Schutte, L. J. Allamandolla, S. A. Sandford, Icarus, 104, 118(1993)

2 H. Cottin, Y. Bénilan, M.-C.Gazeau, F. Raulin, Icarus, 167, 397 (2004)

3 N. Fray, Y. Bénilan, N. Biver, D. Bockelée-Morvan, H. Cottin, J. Crovisier, M.-C. Gazeau, Icarus, 184, 239 (2006)

4 L. Le Roy, G. Briani, C. Briois, H. Cottin, N. Fray, L. Thirkell, G. Poulet, M. Hilchenbach, PSS, 64, 83 (2012)

5 M. P. Bernstein, S. A. Sandford, L. J. Allamandolla, C. Sherwood, M. A. Scharberg, ApJ, 454, 327 (1995)

6 G. M. Muñoz-Caro, W. A. Schutte, A\&A, 412, 121(2003)

7 V. Vinogradoff, A. Rimola, F. Duvernay, G. Danger, P. Theulé, T. Chiavassa, PCCP, 14, 12309 (2012)

8 Vinogradoff V., Fray N., Duvernay F., Briani G., Danger G., Cottin H., Theulé P. and Chiavassa T., A\&A, 551 , A128 (2013)

9 Briani G., Fray N., Cottin H., Benilan Y., Gazeau M.-C. and Perrier S,. Icarus, 226, 541-551 (2013) 